\title{
RFTraffic: a study of passive traffic awareness using emitted RF noise from the vehicles
}

\author{
Yong Ding*, Behnam Banitalebi, Takashi Miyaki and Michael Beigl
}

\begin{abstract}
In this article, a new traffic sensing and monitoring technique is introduced which works based on the emitted RF noise from the vehicles. In comparison with the current traffic sensing systems, our light-weight technique has simpler structure in both terms of hardware and software. An antenna installed to the roadside or the inside of a car receives the signal generated during electrical activity of the vehicles' sub-systems. This signal feeds the feature extraction and classification blocks which recognize different classes of traffic situation in terms of density, flow and location. Different classifiers like naive Bayes, Decision Tree and k-Nearest Neighbor are applied in real-world scenarios and performances for instance of traffic situation detection are reported with higher than $95 \%$. Although the electrical noises of the various vehicles do not have the same statistical characteristics, results from two experiments with an implementation on RF receiver illustrate that our approach is practically feasible for traffic monitoring goals. Due to the acceptable classification results and the differences between the proposed and current traffic monitoring techniques in terms of interfering factors, advantages and disadvantages, we propose it to work in parallel with the current systems to improve the coverage and efficiency of the traffic control network.
\end{abstract}

Keywords: RF noise/signal, traffic sensing, traffic monitoring, traffic awareness, classification

\section{Introduction}

The gradual increase of the traffic demand is saturating the capacity of the transportation network especially in developed countries represented by the EU, USA, and Japan. Due to some reasons like limited possibility of the roads' extension, limited land resources and environmental pollution problem, the development of more efficient traffic management systems has absorbed great attention. Along with the development of ubiquitous computing in different aspects of the everyday life and advances in processing and communication technologies, automated management systems are advancing the human-based ones. Therefore, intelligent transport system (ITS) is one of the key necessities of the future smart cities.

The ITS integrates effectively the technologies like information processing, data communication, electronic sensor, electronic control, and computer processing into the traffic management, in order to establish a comprehensive, real-time transport management system [1],

\footnotetext{
* Correspondence: yong.ding@kit.edu

Department of Informatics, Karlsruhe Institute of Technology (KIT), TecO, Vincenz-Priessnitz-Str. 3, 76131 Karlsruhe, Germany
}

which is accurate and efficient for large-scale applications. Smart transportation elements including intelligent vehicles, intelligent roads and intelligent infrastructures help the drivers efficiently to gain higher level of safety and maneuver capability.

Traffic monitoring is an important part of the ITS. Various road-specific parameters are aggregated to sense the traffic flow. Currently, vision-based methods are widely used in this regard. Cameras together with the advanced image/video processing techniques extract various features about traffic like density and flow or about the individual vehicles like color, shape, length, speed, etc. However dynamic outdoor situations affect their performance [2]. Therefore, vision-based traffic monitoring systems depend more or less on the sensor positioning [3].

We have introduced a new traffic awareness system in our previous work [4]. Because of the electrical activity of various sub-systems like combustion or electrical motors (to derive the pumps or fans), each car emits radio frequency $(\mathrm{RF})$ signals. These signals are different from the environmental noise. This phenomenon enables us to extract the traffic situation information 
from these signals. To achieve this, we design two scenarios (static and dynamic) in this work to install a RF receiver either close to the road or inside the car to aggregate the emitted RF signals from the vehicles. In this work, we evaluate the recognition performance in both static and dynamic scenarios and discuss more about the three classification methods [5]: naive Bayes [6-9], decision tree [10-12] and k-nearest-neighbor $[6,13-15]$, to show the differences (advantage and disadvantage) of various classification algorithms in reality of traffic monitoring. As implemented, these classification methods are applied on the aggregated signal in the computer attached to the RF receiver to classify the traffic situation in both scenarios.

The proposed RF-based traffic awareness system is robust against dynamic illumination or the movement of the background objects. Since it is based on the signals emitted from the cars, this system is passive and in comparison with the other RF-based or vision-based traffic/vehicle monitoring technologies has a simpler structure. Moreover, together with array processing schemes, it is able to sense the traffic density in different directions. Due to its capabilities and advantages, we propose this technique to be applied parallel to or instead of the other traffic sensing systems.

The rest of the article is organized as follows: in the next section, we will review the state of the art in traffic density sensing methods as well as RF-based context recognition applications. Moreover, in this section the effective sub-systems to generate the RF signal of the vehicles are introduced. In Section 3, we focus on the proposed traffic awareness system. For the core functional module of the proposed traffic awareness system, namely the classification module, more discussions about the applied classification methods are described in terms of the implementation in Section 4. As system evaluation, the results of the application of the context recognition algorithms on the aggregated RF signals are represented in Section 5 with respect to different trafficaware scenarios. In Section 6 we discuss about the proposed system, its characteristics and future opportunities. Finally, Section 7 concludes the article.

\section{Related work}

In this section, we offer a brief overview of the state of the art for traffic density sensing approaches and RFbased context recognition, then we introduce relevant sources of the RF signal in the vehicles.

\subsection{Traffic density sensing approaches}

Several methods like push button, magnetic sensors, ranging devices (e.g., RADAR), loop antenna embedded to the road and acoustic- or visual-based systems are used to sense the traffic density. But in this section we focus on the techniques which are capable of being used in the future ITS.

Application of the cameras and images/video processing techniques on the captured data refers to the most popular traffic sensing technique. Depending on the processing capability, various parameters like the vehicle size, speed, color, or the traffic density and flow are detectable. Setchell et al. [3] present a vision-based road-traffic monitoring sensor, which uses an object recognition algorithm to locate vehicles in images of road scenes by searching correspondence space. Another similar work [16] achieves vehicle detection or classification by an iconic object classification scheme for the vision-based traffic sensor system. Based on the existing video-based traffic detecting system, authors [17] present a new solution to segmentation of vehicles from the background, in order to improve the processing speed, the performance during a traffic jam, etc. Other traffic monitoring applications using real-time video/ image tracking are presented for instance based on a virtual line graph for major highway scenarios [18] or based on an active contour model for road intersection scenarios [19]. Low-level image analysis with high-level rule-based reasoning could prove its worth for tracking vehicles in urban traffic scenes [20]. Moreover, video processing techniques are able to track a vehicle even in complex junctions [2].

Nevertheless, vision-based traffic monitoring systems are highly sensitive to the environmental changes: light density and shadows vary continuously or snow, rain and fog limit the vision range of the camera [2]. Most of the image processing techniques are based on the detection of changes in the sequence of images. Therefore, movement of the background objects like trees (because of wind) and people degrades the performance. Moreover, physical movement because of the wind or other parameters may degrade the monitoring performance.

By development of the inter-vehicle communication capabilities [21] in the vehicles, traffic sensing techniques are proposed based on car-to-car communication (C2C) [22]. But such methods need the collaboration of each unit of the vehicles. However, there is no guarantee about the performance of such systems due to lack or defection of the proper communication features (old vehicles) or due to deactivation of the $\mathrm{C} 2 \mathrm{C}$ communication subsystems by the drivers.

\subsection{RF-based context recognition}

Context awareness is starting to play an increasingly important role in different areas of pervasive computing, especially in recognition applications, which are able to adapt their operations to the current situational context without explicit user intervention [23]. Context, according to Dey and Abowd [24], is any information that can 
be used to characterize the situation of an entity, where an entity is a person, place or object that is considered relevant to the interaction between a user and an application.

The most researched context recognition scenarios are often cited as applications of activity recognition, situation recognition, motion detection, etc., which usually utilize wireless sensor nodes equipped with various sensors to detect situation. Due to several constraints of wireless sensing with sensors, like power consumption, communication bandwidth, and deployment costs, now researchers have begun investigation of different features in RF propagation for the purpose of context recognition [25]. The RF signal is generated by nearly every electronic device [26], such as mobile phones, notebooks, watches, motors, etc., so the additional cost for using this signal in a recognition application is considerably low.

Woyach et al. [27] present a sensor-less sensing approach to detect the motion of objects based on received signal strength measurements on MICAz nodes, which illustrates that the motion of objects with respect to the velocity can be estimated by means of a signal strength pattern analysis. Another similar work [28] achieves WiFi-based motion detection by analyzing spectral characteristics of WLAN radio signal strength and its fluctuations. Fluctuations in GSM signal strength have also been used for detecting user mobility $[29,30]$. Besides observing the absolute RSSI values like [27], Lee et al. [25] employ the fluctuation counting in RSSI values on a restricted frequency band for motion detection. Other classification applications, such as electrical event detection in a home environment through sensing the electromagnetic interference [31] and room situation classification based on RF-channel measurements [32], show also a great potential of RF signal features for activity recognition.

We note that in most of the previous RF-based context recognition systems, a RF signal is transmitted through the target and receiver uses the shape and strength of the reflections for classification. Our method for detecting traffic situation proposes to extract information about for instance traffic density by using only emitted RF signals from the vehicles passing by.

\subsection{Sources of RF noise in the vehicles}

Modern vehicles are composed of various electronic components like: electric ignition, motors to drive different pumps (oil, water or fuel) or fans, other sub-systems like communication sub-systems (radio receiver/transmitters), microprocessors, sensors, entertainment facilities and wires that route the signals among the electronic sub-systems. Some of these sub-systems are expected to have RF emission with specific patterns, e.g., during ignition procedure, relatively strong impulses are generated or a periodic behavior from the electric motors is expected. Despite of the complexity and variety of the emitted RF signal from the vehicles, this signal contains information about the vehicles' situation. For instance in $[33,34]$, RF emission is used to detect various car models, but in the isolated test environment.

\section{Proposed traffic awareness system}

Our proposed traffic awareness system is designed to investigate traffic information extraction on the road intersection. While most traffic congestion or traffic flow estimation approaches rely on only sensory data of observed road segments [35] and do not consider other surrounding context. Our approach focuses on simply utilizing emitted RF signals from the vehicles to discover current traffic situation context instead of relying on only sensory data of observed road segments. The context that is investigated in this article for the traffic flow or situation estimation will be defined in Section 3.3 depending on the scenarios.

\subsection{Feasibility study}

In this section, we will illustrate some features of the RF noises from the vehicles based on the first dataset. Firstly we calculate the mean value of all captured data, which correspond to either environment or cars moving by. It is easy to see in Figure 1, the mean value of environment situation is averagely greater than the mean value of carmoving situation. Thanks to the different mean value levels (see magenta and green dash lines in Figure 1), the mean value of RF noises can be used as a classification feature to distinguish car movement from the environment.

Then we investigate the FFT amplitude of the RF noises. Figure 2 shows two FFT curves corresponding to the environment and cars without movement respectively. In our work, the cars without movement refer to the cars, which are totally stopped and subsequently switch off their engines. The different curve progressions in the figure prove clearly that the FFT amplitude of the RF noises can be considered as another feature for classifying different traffic situations.

Through such a simple feasibility study, we believe that the RF noises from the vehicles can be used as the only information source for traffic sensing.

\subsection{Experimental setup}

\subsubsection{Static scenario}

We used a USRP ${ }^{a}$ software radio equipped with a 2.4 $\mathrm{GHz}$ transceiver board (RFX2400) which is installed to the roadside and a VERT2450 antenna module with $3 \mathrm{dBi}$ antenna gain is used to receive the emitted RF signal from the vehicles. We tested the emitted signals in limited frequency bands, but the signals at $2.4 \mathrm{GHz}$ 


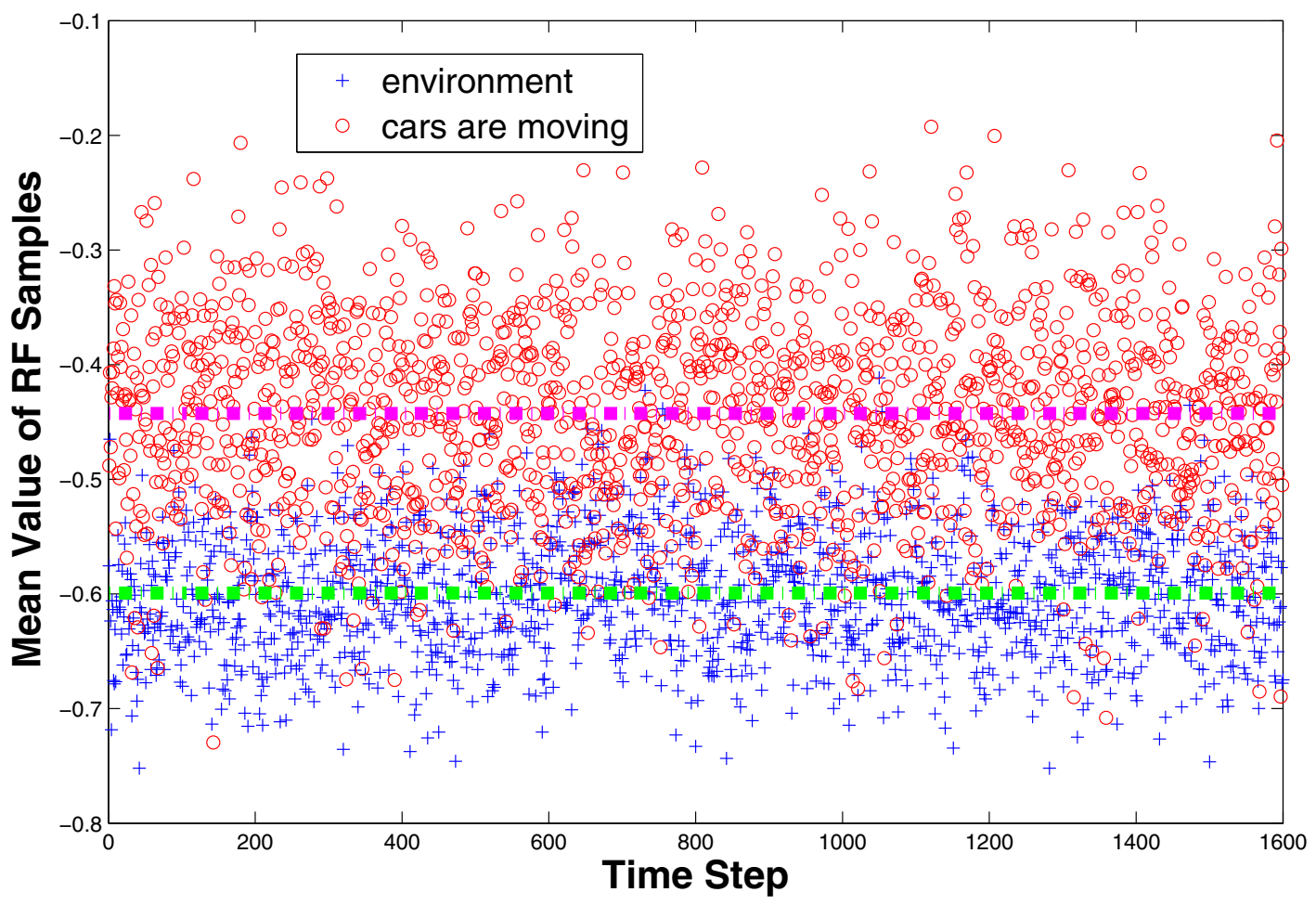

Figure 1 The Mean Value of RF signal features different behaviors in different situation.

matched to our application more (To minimize the set up, higher frequencies are considered). A laptop PC is connected to the USRP which is responsible for data acquisition and application of the feature extraction and classification algorithms. The basic illustration of this experimental setup is depicted in Figure 3.

Furthermore, the USRP device is configured to listen to the channel continuously while calculating the features used for classification at a sampling rate of 320,000 samples/second. As the power supply for the USRP device in our prototype is a car battery, a preprocessing step is designed for extracting the environmental context without any traffic but this power supply car, in order to avoid further interference to the received signal and so achieve more accurate classification results.

\subsubsection{Dynamic scenario}

For a dynamic scenario, we accomplished the measurement using a USRP software radio installed inside the car, which is equipped with a $2.4 \mathrm{GHz}$ transceiver board (RFX2400) and a $900 \mathrm{MHz}$ transceiver board (RFX900) respectively. VERT2450 and VERT900 antenna modules with $3 \mathrm{dBi}$ antenna gain are used respectively to receive the emitted RF signal from the vehicles.
As in the static scenario, a laptop PC is connected to the USRP which is responsible for data acquisition and application of the feature extraction and classification algorithms. The basic illustration of this experimental setup is depicted in Figure 4. Furthermore, the USRP device and the preprocessing step are configured as well as in Section 3.2.1.

\subsection{Context recognition}

We study the feasibility to obtain an awareness on traffic situations in experimental instrumentation with only an USRP software radio as described in Section 3.2. In general, the proposed approach refers to a context recognition system for traffic awareness of road segments and vehicular location, which consists of four functional modules illustrated in Figure 5:

- Data acquisition: The first step in any data analysis task is naturally data collecting, so is in our traffic awareness scenario as well. As described before, the data acquisition for the proposed traffic awareness system is accomplished only through a light-weight RF signals received with an USRP node at $2.4 \mathrm{GHz}$ 


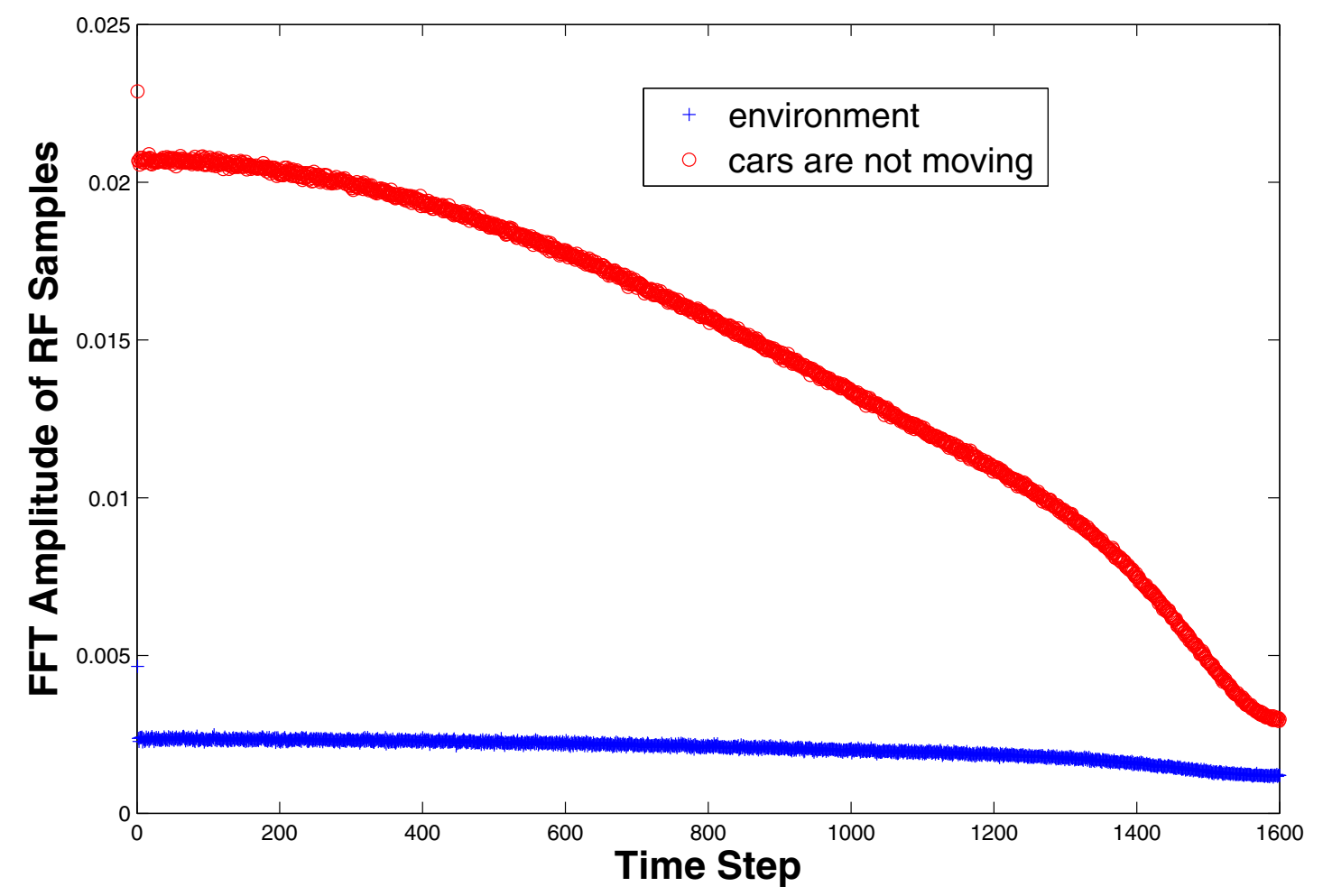

Figure 2 The FFT Amplitude of RF signal features different behaviors in different situation.

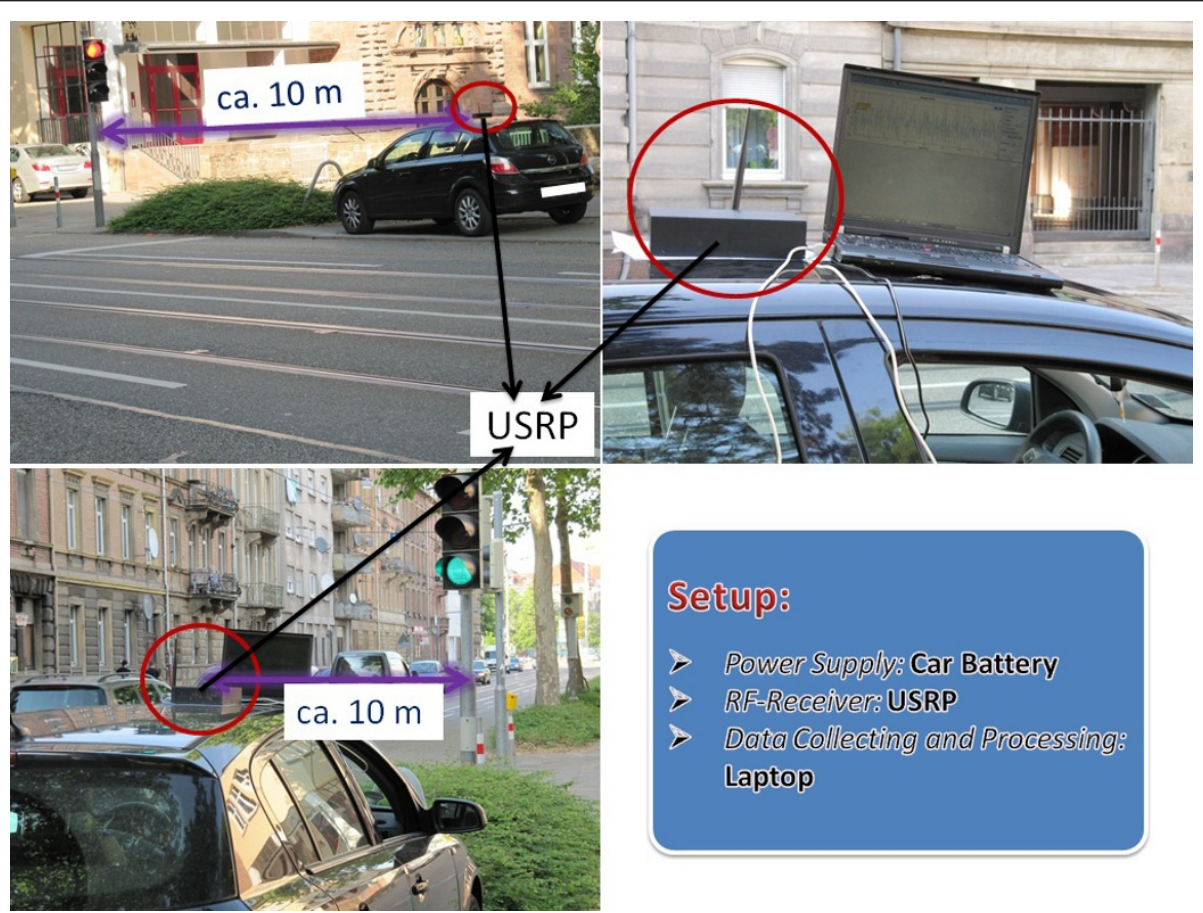

Figure 3 Experimental setup for the proposed traffic awareness system in the static situation. 


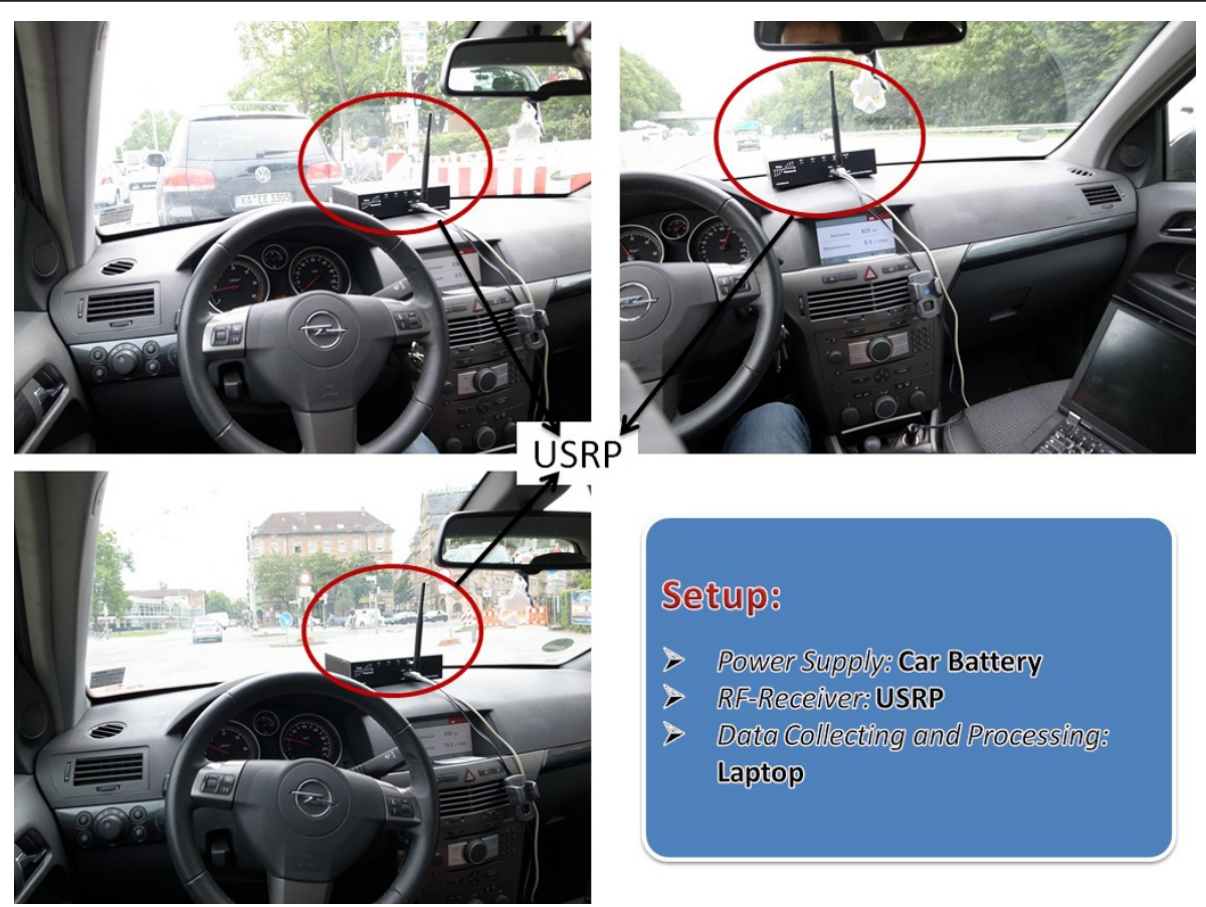

Figure 4 Experimental setup for the proposed traffic awareness system in the dynamic situation.

or $900 \mathrm{MHz}$ instead of conventional sensor-based sensing.

- Feature extraction: The next step is to derive features from the raw RF measurements using statistical and signal processing techniques. To feed the next module (classification), we sampled the mean value, standard deviation, root mean square (RMS) and FFT amplitude of the received signals.

- Classification: After feature extraction, a feature vector is forwarded to the classification process in both learning phase and real-time estimation phase. As illustrated in the system schema (Figure 5), we employ naive Bayes (probabilistic classifier), decision tree (predictive model) and k-NN (k-nearest neighbor algorithm: instance-based learning) for the classification module and compare the results.
- Application: To ease the further processing of the classified contexts for traffic awareness, certain highlevel contexts can be interpreted based on the classified low-level traffic contexts and then integrated into the existing traffic sensing applications (e.g., traffic density, traffic jam/flow and vehicular location).

\subsubsection{Static scenario}

The precondition of a real-time traffic awareness is the predefined context attributes for the traffic situation estimation, which is the only step in the proposed architecture that requires user interaction in the proposed architecture. Correlation of the context attributes to the observed road segment can not be neglected. So we limit the definition of context attributes only for the traffic density with respect to the traffic light as follows,

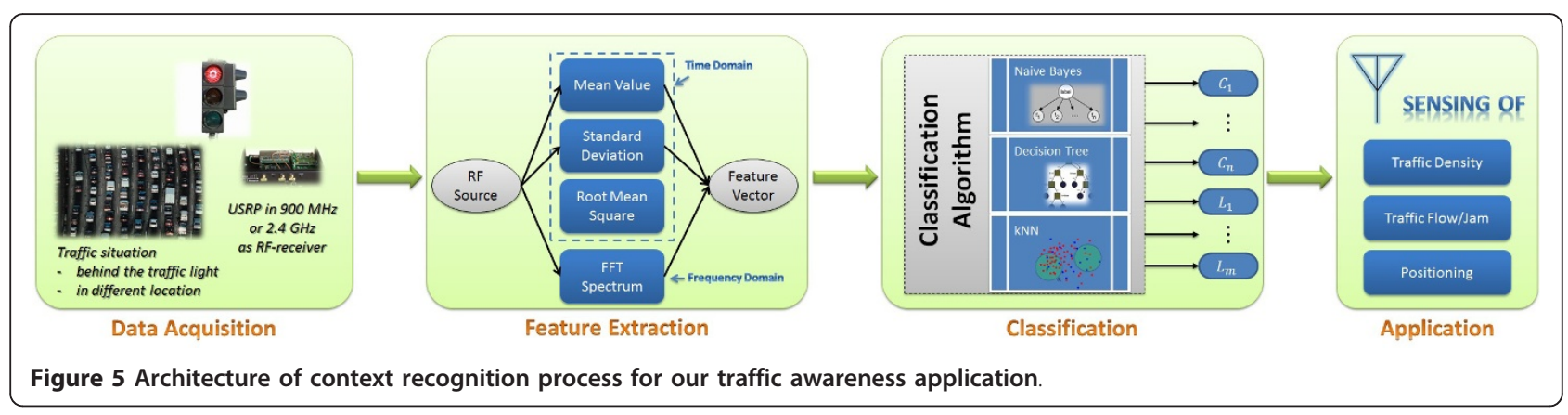




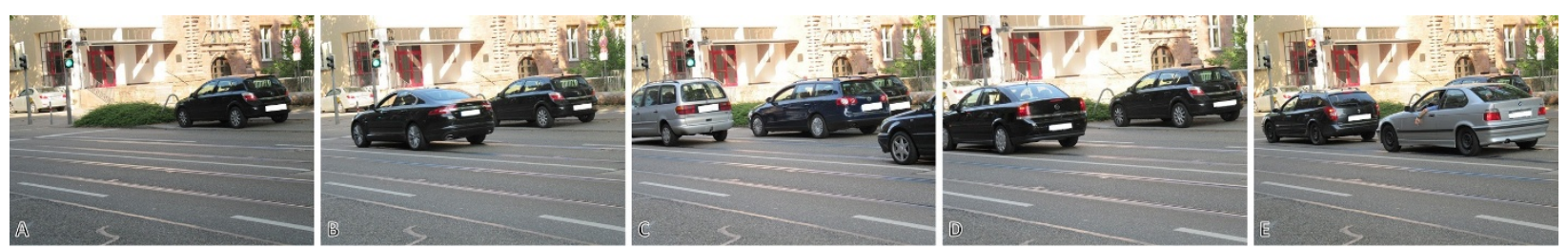

Figure 6 Five different traffic situation scenarios behind a traffic light. (a) Environment without any traffic flow; (b) smooth traffic flow with only one car driving by; (c) smooth traffic flow with many cars driving by; (d) only one car behind the red traffic light; (e) many cars behind the red traffic light.

which are five different traffic density situations demonstrated in Figure 6.

- Environment $\left(C_{1}\right)$ : which means no traffic flow/jam at all, see Figure 6a.

- Smooth traffic with one car $\left(C_{2}\right)$ : which means only few cars drive by the green traffic light at that moment and corresponds to no congestion, see Figure $6 \mathrm{~b}$.

- Smooth traffic with many cars $\left(C_{3}\right)$ : which means lots of cars drive by the green traffic light at that moment and corresponds to low congestion, see Figure $6 \mathrm{c}$.

- One car stopped $\left(C_{4}\right)$ : which means only few cars wait right now behind the red traffic light and corresponds to medium congestion, see Figure $6 \mathrm{~d}$.

- Many cars stopped $\left(C_{5}\right)$ : which means lots of cars wait right now behind the red traffic light and corresponds to high congestion, see Figure $6 \mathrm{e}$.

\subsubsection{Dynamic scenario}

For the dynamic scenario, in which the RF receiver is installed inside a moving car, we limit the definition of context attributes only for the vehicular location with respect to the velocity of the car as follows, which are 3 different vehicular location scenarios demonstrated in Figure 7.

- Start to drive $\left(L_{1}\right)$ : which refers to velocity at $0 \mathrm{~km} /$ $\mathrm{h}$, see Figure $7 \mathrm{a}$.
- Driving in the urban traffic $\left(L_{2}\right)$ : which refers to velocity between 30 and $70 \mathrm{~km} / \mathrm{h}$, see Figure $7 \mathrm{~b}$.

- Driving in the highway traffic $\left(L_{3}\right)$ : which refers to velocity more than $100 \mathrm{~km} / \mathrm{h}$, see Figure 7c.

\section{Classification methods}

For both scenarios, we implement and evaluate several standard features of the RF signals in different representational domains, i.e., time and spectral domain. The features generated are mean, standard deviation, RMS, and FFT amplitude, since these were often cited as being the most decisive for classification applications [36-40]. Since this work concerns primarily the feasibility of traffic monitoring only based on RF receiving, so a feature selection step for more accurate classification is not a part of this work.

The MATLAB data mining toolboxes [41] are selected for traffic situation recognition because of the portability and domain specific representations of MATLAB programme [42], and the simple efficient interface between the MATLAB signal processing and the USRP receiving platform [43-47].

\subsection{Decision tree}

C4.5 decision tree algorithm [48] is extremely useful supervised learning tools in the field of data mining. In our work, the decision tree algorithm was used for classification due to its prevalence in the literature of

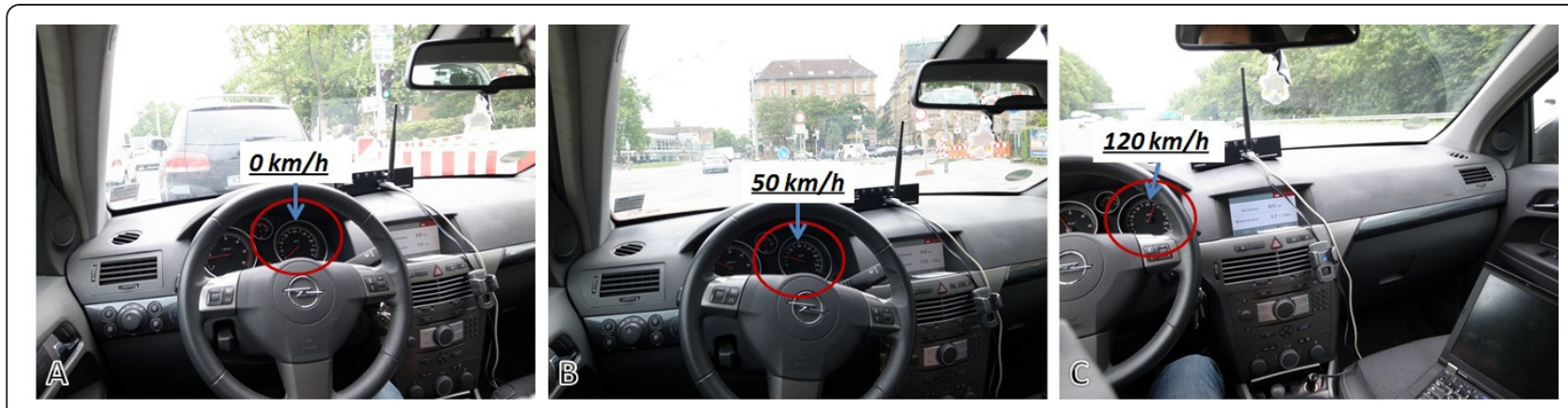

Figure 7 Three different vehicular location scenarios. (a) Car just starts to drive; (b) car drives in the urban traffic; (c) car drives in the highway traffic. 
sensor-based activity recognition [36,39,49-51]. The classification process based on decision tree algorithm starts at the root of the tree and proceeds to a leaf, which indicates the classification output [52]. Each node on the path (a disjunction of test to make the final decision) to a leaf includes a decision which path further to proceed.

\subsection{Naive Bayes}

The naive Bayes approach has several advantages like its simplicity and transparency, which is the simplest form of a Bayesian network [53]. Another advantage of the naive Bayes classifier is that it only requires a small amount of training data to estimate the parameters (means and variances of the variables) necessary for classification. In naive Bayes classification, conditional independence of the feature values $f_{i}$ of feature vector $F$ is assumed. Accordingly, the probability of $F$ given a certain class $c_{i}$ is calculated by multiplying the the probabilities of each $f_{i}$. It is important to know that the posterior probability of feature values is proportional to a certain class prior $p\left(c_{i}\right)$ multiplied by the product of the appropriate (independent) likelihoods conditioned on $\mathrm{c}_{\mathrm{i}}$. For this classification work, we implement a Gaussian for each class in static $\left(C_{1}\right.$ to $\left.C_{5}\right)$ and dynamic $\left(L_{1}\right.$ to $\left.L_{3}\right)$ scenario respectively from the training dataset. Furthermore, we choose the (MAP) decision rule to obtain the final decision. The corresponding classifier is the function classify defined as follows:

$$
\operatorname{classify}\left(f_{1}, \ldots, f_{n}\right)=\underset{c}{\operatorname{argmax}} p(C=c) \prod_{n}^{i=1} p\left(F_{i}=f_{i} \mid C=c\right) .
$$

Whereat the $C$ and $F_{i}$ are random variable of class and feature respectively.

\section{$4.3 k$-nearest neighbor}

The $k$-NN approach [54] is a method for classifying objects based on closest training examples in the feature space. In order to provide a comparison between standard classification algorithms [55,56], we also implemented and evaluated a $k$-NN classifier with $k$ chosen as 1 or $20 \%$ of the training dataset. Proper choice of $k$ depends on the data, since smaller $k$ leads to higher variance, which means less stable, and larger $k$ leads to higher bias, which means less precise. The in this work applied $k$-NN algorithm functions as follows:

(1) Calculate Euclidean distance of test vector to all training vectors that were sampled.

(2) Pick $k$ closest training vectors according to the above distance metric.

(3) Classify the predicted class by majority vote of the $k$ closest training vectors.
(4) Improve the class through multiplying an average weighted by inverse distance.

\section{Evaluation}

The first experiment was conducted for a road segment with two lanes in each direction. In the experiment we attempted to derive the five context classes of the traffic density described in Section 3.3.1. To gather meaningful performance data, we must firstly determine the requirements for the dataset capturing. On the one hand, with respect to the average duration of the red light (ca. 15 $\mathrm{s})$, we restrict the size of each dataset for $10 \mathrm{~s}$, so that these five context classes can be distinguished from each other without temporal overlap. On the other hand, in order to differ the dataset of red traffic light scenarios from green ones, we set a stop time for the data gathering during the red light just when the red light turns to green.

In the second experiment we attempted to derive the three context classes of the vehicular location described in Section 3.3.2. The same as in the first experiment, we must firstly determine the requirements for the dataset capturing. For each class of the vehicular location, e.g., Start $\left(L_{1}\right)$, Urban $\left(L_{2}\right)$ and Highway $\left(L_{3}\right)$, we collected five different datasets respectively. And each dataset has a size for more than $1 \mathrm{~min}$.

For each classification we set a window size of 2,000 samples in the feature extraction, i.e., for training, each dataset is fetched for 1,600 feature values. In general, the traffic awareness system is now evaluated off-line. As mentioned in Section 3.3, we adopt naive Bayes, decision tree and $k$-NN for our situation classification module and compare the results in terms of the accuracy and confusion matrix. To avoid any bias caused by the particular sampling chosen for training and testing, we validate all three classification algorithms with a stratified 10-fold cross-validation, through which the dataset is partitioned randomly into ten subsamples. Each subsample is held out in turn for testing and the remaining nine subsamples are used as training data [57].

The accuracy of the traffic density awareness using different classification algorithms is shown in Tables 1 , 2 , and 3 , respectively. We observe that the average accuracy for the situation awareness with all three classifiers is rather high, which is over 95\%. From the point of view of the results, especially the first four situation classes, i.e., $C_{1}, C_{2}, C_{3}$, and $C_{4}$ could be detected very well with an average false negative rate of 1.4, 2.0, 6.2, and $2.8 \%$ respectively. As we see, the fifth class, $C_{5}$, i.e., "any cars stopped", whose recognition rate is still considerably high with an average accuracy of $87.6 \%$. But compared to the other four classes, the average classification accuracy of $C_{5}$ drops about $10 \%$. While a loss in 
Table 1 Classification accuracy achieved with naive Bayes classifier for traffic density awareness using one USRP device in the setting depicted in Figure 3

\begin{tabular}{llllll}
\hline & \multicolumn{5}{c}{ Predicted (\%) } \\
\cline { 2 - 6 } $\mathrm{C}_{\mathbf{1}}$ & $\mathrm{C}_{\mathbf{2}}$ & $\mathrm{C}_{\mathbf{3}}$ & $\mathrm{C}_{\mathbf{4}}$ & $\mathrm{C}_{\mathbf{5}}$ \\
\hline$C_{1}$ & $\mathbf{9 8 . 1}$ & 1.6 & 0.3 & 0 & 0 \\
$C_{2}$ & 1.2 & $\mathbf{9 8 . 7}$ & 0.1 & 0 & 0 \\
$C_{3}$ & 0.6 & 4.7 & $\mathbf{9 4 . 7}$ & 0 & 0 \\
$C_{4}$ & 0 & 0 & 0 & $\mathbf{9 9 . 9}$ & 0.1 \\
$C_{5}$ & 0 & 0 & 0 & 16.7 & $\mathbf{8 3 . 3}$ \\
\hline
\end{tabular}

Note: the bold values indicate the true positive rate of each class. The same indication applies to Tables 2 through 9 as well.

accuracy for the class of "many cars stopped" was expected due to the RF signal strength and receiving range.

The accuracy of the vehicular location awareness using different classification algorithms is shown in Tables 4, 5, and 6 for RF receiving at $900 \mathrm{MHz}$, Tables 7, 8, and 9 for RF receiving at $2.4 \mathrm{GHz}$. We notice that the average accuracy for the location awareness with all three classifiers and at both frequencies is not bad, which reaches ca. $89 \%$. And at both frequencies $(900$ $\mathrm{MHz}$ and $2.4 \mathrm{GHz}$ ), the urban class is well detected with an average false negative rate of 5.7 and $4.6 \%$, respectively. From the point of view of the accuracy results in Tables $4,5,6,7,8$, and 9, there is no significant dissimilarity between the classification at $900 \mathrm{MHz}$ and $2.4 \mathrm{GHz}$.

As mentioned before, we provide mean value, standard deviation, RMS, and FFT amplitude of the received signals as features for the classification process, the last two contributed more. Figure 8 shows finally the distribution of five defined traffic density situations with respect to for instance two features of mean value and FFT amplitude after classification using decision tree algorithm, while Figure 9 depicts the distribution of three predefined vehicular location classes at different frequency bands by way of comparison. We observe that particularly the urban and highway scenarios have explicit difference in terms of distribution behavior of RF features.

Table 2 Classification accuracy achieved with decision tree classifier for traffic density awareness using one USRP device in the setting depicted in Figure 3

\begin{tabular}{llllll}
\hline & \multicolumn{5}{c}{ Predicted (\%) } \\
\cline { 2 - 6 } Actual & $\mathbf{C}_{\mathbf{1}}$ & $\mathbf{C}_{\mathbf{2}}$ & $\mathbf{C}_{\mathbf{3}}$ & $\mathbf{C}_{\mathbf{4}}$ & $\mathbf{C}_{\mathbf{5}}$ \\
\hline$C_{1}$ & $\mathbf{9 9 . 3}$ & 0.6 & 0.1 & 0 & 0 \\
$C_{2}$ & 0.7 & $\mathbf{9 8 . 9}$ & 0.4 & 0 & 0 \\
$C_{3}$ & 0.5 & 1.9 & $\mathbf{9 7 . 6}$ & 0 & 0 \\
$C_{4}$ & 0 & 0 & 0 & $\mathbf{9 8 . 1}$ & 1.9 \\
$C_{5}$ & 0 & 0 & 0 & 2.1 & $\mathbf{9 7 . 9}$ \\
\hline
\end{tabular}

Table 3 Classification accuracy achieved with $k$-NN ( $k=$ 1) classifier for traffic density awareness using one USRP device in the setting depicted in Figure 3

\begin{tabular}{llllll}
\hline & \multicolumn{5}{c}{ Predicted (\%) } \\
\cline { 2 - 6 } Actual & $\mathrm{C}_{\mathbf{1}}$ & $\mathrm{C}_{\mathbf{2}}$ & $\mathrm{C}_{\mathbf{3}}$ & $\mathrm{C}_{\mathbf{4}}$ & $\mathrm{C}_{\mathbf{5}}$ \\
\hline$C_{1}$ & $\mathbf{9 8 . 5}$ & 1.4 & 0.1 & 0 & 0 \\
$C_{2}$ & 1.5 & $\mathbf{9 6 . 4}$ & 2.1 & 0 & 0 \\
$C_{3}$ & 0.8 & 10 & $\mathbf{8 9 . 2}$ & 0 & 0 \\
$C_{4}$ & 0 & 0 & 0 & $\mathbf{9 3 . 6}$ & 6.4 \\
$C_{5}$ & 0 & 0 & 0 & 18.4 & $\mathbf{8 1 . 6}$ \\
\hline
\end{tabular}

\section{Discussion}

\subsection{Classification performance}

In general, the decision tree performed better compared to the other classifiers (naive Bayes and $k$-NN). As shown in Tables 2, 5, and 8, the decision tree achieved rather high average classification rate of 98.4, 94.5, and $94.3 \%$ in both scenarios with different frequencies respectively. The results (Tables 1, 2, and 3) of the accuracy of the traffic density awareness indicate that the decision tree only slightly outperformed the naive Bayes (94.9\%) and $k$-NN (91.9\%) in this scenario with a classification rate of $98.4 \%$ on average. The accuracy of the vehicular location awareness at either $900 \mathrm{MHz}$ (Tables 4, 5, and 6) or 2.4 GHz (Tables 7, 8, and 9) indicates that a 9 and $6 \%$ decrease in overall system classification rates, respectively for naive Bayes and $k-\mathrm{NN}$.

Comparing feature extraction and classification time (see Table 10) it is worth pointing out that the major percentage of the total time of the feature-based classification process is dependent on the classification algorithm. The percentage of the decision tree classification task does not exceed $7.2 \%$ of the complete processing time, while $k-\mathrm{NN}$ and naive Bayes require an upper bound of 65.4 and $72.5 \%$ for the classification task.

A motion recognition work of Yang [49] concluded that $k$-NN can achieve good performance for selected time-domain magnitude features; but decision tree is found to achieve the best performance among four different static classifiers with acceptable computational complexity, while vertical/horizontal features have better performance than magnitude features. As shown in

Table 4 Classification accuracy achieved with naive Bayes classifier for vehicular location awareness using one USRP device at $900 \mathrm{MHz}$ in the setting depicted in Figure 4

\begin{tabular}{llll}
\hline & \multicolumn{3}{c}{ Predicted (\%) } \\
\cline { 2 - 4 } Actual & Start $\left(\mathrm{L}_{\mathbf{1}}\right)$ & Urban $\left(\mathrm{L}_{\mathbf{2}}\right)$ & Highway $\left(\mathrm{L}_{3}\right)$ \\
\hline Start $\left(\mathrm{L}_{1}\right)$ & $\mathbf{8 3 . 3}$ & 12.9 & 3.8 \\
Urban $\left(L_{2}\right)$ & 0 & $\mathbf{9 9 . 2}$ & 0.8 \\
Highway $\left(L_{3}\right)$ & 10.1 & 14.7 & $\mathbf{7 5 . 2}$ \\
\hline
\end{tabular}


Table 5 Classification accuracy achieved with decision tree classifier for vehicular location awareness using one USRP device at $900 \mathrm{MHz}$ in the setting depicted in Figure 4

\begin{tabular}{llll}
\hline & \multicolumn{3}{c}{ Predicted (\%) } \\
\cline { 2 - 4 } Actual & Start $\left(\mathrm{L}_{\mathbf{1}}\right)$ & Urban $\left(\mathrm{L}_{\mathbf{2}}\right)$ & Highway $\left(\mathrm{L}_{\mathbf{3}}\right)$ \\
\hline Start $\left(\mathrm{L}_{1}\right)$ & $\mathbf{9 4 . 4}$ & 3.5 & 2.1 \\
Urban $\left(\mathrm{L}_{2}\right)$ & 2.0 & $\mathbf{9 5 . 9}$ & 2.1 \\
Highway $\left(L_{3}\right)$ & 4.3 & 2.5 & $\mathbf{9 3 . 2}$ \\
\hline
\end{tabular}

Section 3.1, the frequency-domain feature (FFT) can better distinguish situational RF signals compared to time-domain features. Therefore, the experimental results with both respect to classification accuracy and processing time show that the decision tree algorithm has the best performance for classification tasks in our application scenarios. But decision tree must not always achieve the best classification performance, especially in the case of sensor-based classification, like Fischer et al. [14] investigated occupant recognition in parked cars, in which the best results are achieved by the k-NN algorithm. In order to choose an adequate classification algorithm for a certain application, the signal property (in case of not only sensor-based but also sensorless scenarios), extracted feature property and the number of classes, must be considered as well.

\subsection{System characteristics and future opportunities}

Simplicity is one of the positive aspects of our proposed technique. At hardware part, there is only one receiver together with the antenna whereas in the software part, the applied classification algorithms are relatively simpler than those used in vision-based techniques. Although limited classes of traffic density and flow, as well as vehicular locations are detectable, but as seen in Tables 1, 2, 3, 4, 5, 6, 7, 8, and 9, the performance is relatively high. This simplicity which directly affects the price, would be beneficial to more expansion of the traffic sensing and monitoring network. Moreover, the proposed method is more suitable for miniaturized applications like covert traffic monitoring.

Table 6 Classification accuracy achieved with $k$-NN ( $k=$ 1) classifier for vehicular location awareness using one USRP device at $900 \mathrm{MHz}$ in the setting depicted in Figure 4

\begin{tabular}{llll}
\hline & \multicolumn{3}{c}{ Predicted $(\%)$} \\
\cline { 2 - 4 } Actual & Start $\left(\mathrm{L}_{\mathbf{1}}\right)$ & Urban $\left(\mathrm{L}_{\mathbf{2}}\right)$ & Highway $\left(\mathrm{L}_{\mathbf{3}}\right)$ \\
\hline Start $\left(\mathrm{L}_{1}\right)$ & $\mathbf{8 2 . 0}$ & 14.4 & 3.6 \\
Urban $\left(L_{2}\right)$ & 8.4 & $\mathbf{8 7 . 8}$ & 3.8 \\
Highway $\left(L_{3}\right)$ & 7.5 & 5.9 & $\mathbf{8 6 . 6}$ \\
\hline
\end{tabular}

Table 7 Classification accuracy achieved with naive Bayes classifier for vehicular location awareness using one USRP device at $2.4 \mathrm{GHz}$ in the setting depicted in Figure 4

\begin{tabular}{llll}
\hline & \multicolumn{3}{c}{ Predicted (\%) } \\
\cline { 2 - 4 } Actual & Start $\left(\mathrm{L}_{\mathbf{1}}\right)$ & Urban $\left(\mathrm{L}_{\mathbf{2}}\right)$ & Highway $\left(\mathrm{L}_{3}\right)$ \\
\hline Start $\left(\mathrm{L}_{1}\right)$ & $\mathbf{7 8 . 8}$ & 0 & 21.2 \\
Urban $\left(\mathrm{L}_{2}\right)$ & 0 & $\mathbf{9 5 . 3}$ & 4.7 \\
Highway $\left(L_{3}\right)$ & 18.5 & 0.2 & $\mathbf{8 1 . 3}$ \\
\hline
\end{tabular}

The performance of the vision-based systems is highly dependent on the light density and the background objects. Although RF signals are also affected by the transmission channel and interfering signals, we did the tests both in daytime (between 14:00 and 17:00) to have relatively worse case of interference level. More accurate tests to compare the variation of the negative transmission channel effects by time on the classification performance are needed.

The main goal of this article is to introduce a new traffic monitoring technique, so that the static setup which means installation of a RF-receiver close to the roadside, may represent more benefits of our RFTraffic system. Due to its performance, flexibility, and robustness, the proposed technique has lots of potential applications which are under research. There are various kinds of antennae with different patterns [58], most of them are applicable to receive the emitted signals from the cars. It enables our traffic sensing system to sense the traffic situation at a certain direction. Moreover, together with array processing schemes [59], the proposed system can change its pattern by modification of the phase shifts of the antenna elements or to process the signals of more than one direction at the same time. Multiple antennae are also applicable in another form. Each receiver can sense the traffic density of a limited area around itself due to its limited reception capability, i.e., the proposed traffic monitoring has limited range depending on the receiver sensitivity. Installation of the multiple antennae along the street behind the traffic light allows us to figure out the exact length of the traffic jam.

Table 8 Classification accuracy achieved with decision tree classifier for vehicular location awareness using one USRP device at $2.4 \mathrm{GHz}$ in the setting depicted in Figure 4

\begin{tabular}{llll}
\hline & \multicolumn{3}{c}{ Predicted (\%) } \\
\cline { 2 - 4 } Actual & Start $\left(\mathbf{L}_{\mathbf{1}}\right)$ & Urban $\left(\mathrm{L}_{\mathbf{2}}\right)$ & Highway $\left(\mathrm{L}_{\mathbf{3}}\right)$ \\
\hline Start $\left(\mathrm{L}_{1}\right)$ & $\mathbf{9 2 . 8}$ & 0.3 & 6.9 \\
Urban $\left(\mathrm{L}_{2}\right)$ & 0.3 & $\mathbf{9 8 . 6}$ & 1.1 \\
Highway $\left(\mathrm{L}_{3}\right)$ & 7.9 & 0.6 & $\mathbf{9 1 . 5}$ \\
\hline
\end{tabular}


Table 9 Classification accuracy achieved with $k$-NN ( $k=$ 1) classifier for vehicular location awareness using one USRP device at $2.4 \mathrm{GHz}$ in the setting depicted in Figure 4

\begin{tabular}{llll}
\hline & \multicolumn{3}{c}{ Predicted (\%) } \\
\cline { 2 - 4 } Actual & Start $\left(\mathrm{L}_{\mathbf{1}}\right)$ & Urban $\left(\mathrm{L}_{\mathbf{2}}\right)$ & Highway $\left(\mathrm{L}_{\mathbf{3}}\right)$ \\
\hline Start $\left(\mathrm{L}_{1}\right)$ & $\mathbf{8 5 . 8}$ & 0.4 & 13.8 \\
Urban $\left(L_{2}\right)$ & 1.3 & $\mathbf{9 2 . 3}$ & 6.4 \\
Highway $\left(L_{3}\right)$ & 12.6 & 4.6 & $\mathbf{8 2 . 8}$ \\
\hline
\end{tabular}

Other forms of classification of the vehicles like based on their dimensions: motorcycle, car, van, bus, or based on their location: city or highway (primary tests show its feasibility) are also possible. Various location aware applications can be then defined based on this possibility.

Comparison of proposed traffic monitoring technique with current video-based ones shows that due to their independence in terms of interfering or distorting factors, capabilities, advantages, and disadvantages, as well as the potential extensions of the proposed system, it can be used in parallel with the current traffic monitoring systems complementarily to cover their drawbacks. Due to the complexity, high sensitivity to the position, angle, and financial issues, to cover the entire transportation network with the visual-based systems is not feasible. Moreover, the variation of the weather condition affects the performance of traffic cameras severely. On the contrary, weather changes have relatively less negative effects on the RF signals. Besides, our proposed RFTraffic system has less complexity (both in terms of processing algorithms and hardware) and is relatively robust against small changes.

RFTraffic may realize other potential applications. In terms of traffic monitoring, the equipment of RFTraffic with array antennae could enable it to figure out the traffic situation of different directions. This feature will accelerate the traffic monitoring process. RFTraffic can also be used inside the vehicles as part of the navigation system, e.g., to sense the traffic density on different sides of the vehicle especially in case of limited visibility like foggy or dusty situations. Furthermore, RFTraffic can also be considered as an activity awareness system to manage the vehicular sub-systems depending on the driving mode. For instance, cellphones can take advantage of RFTraffic to divert coming calls to the voice-box and avoid interfering of the driver during driving.

\section{Conclusion}

Traffic situation recognition is one important component for ITS. In this article, we represented the feasibility of a new traffic awareness technique. It uses the RF signals emitted from the cars. The proposed technique has a

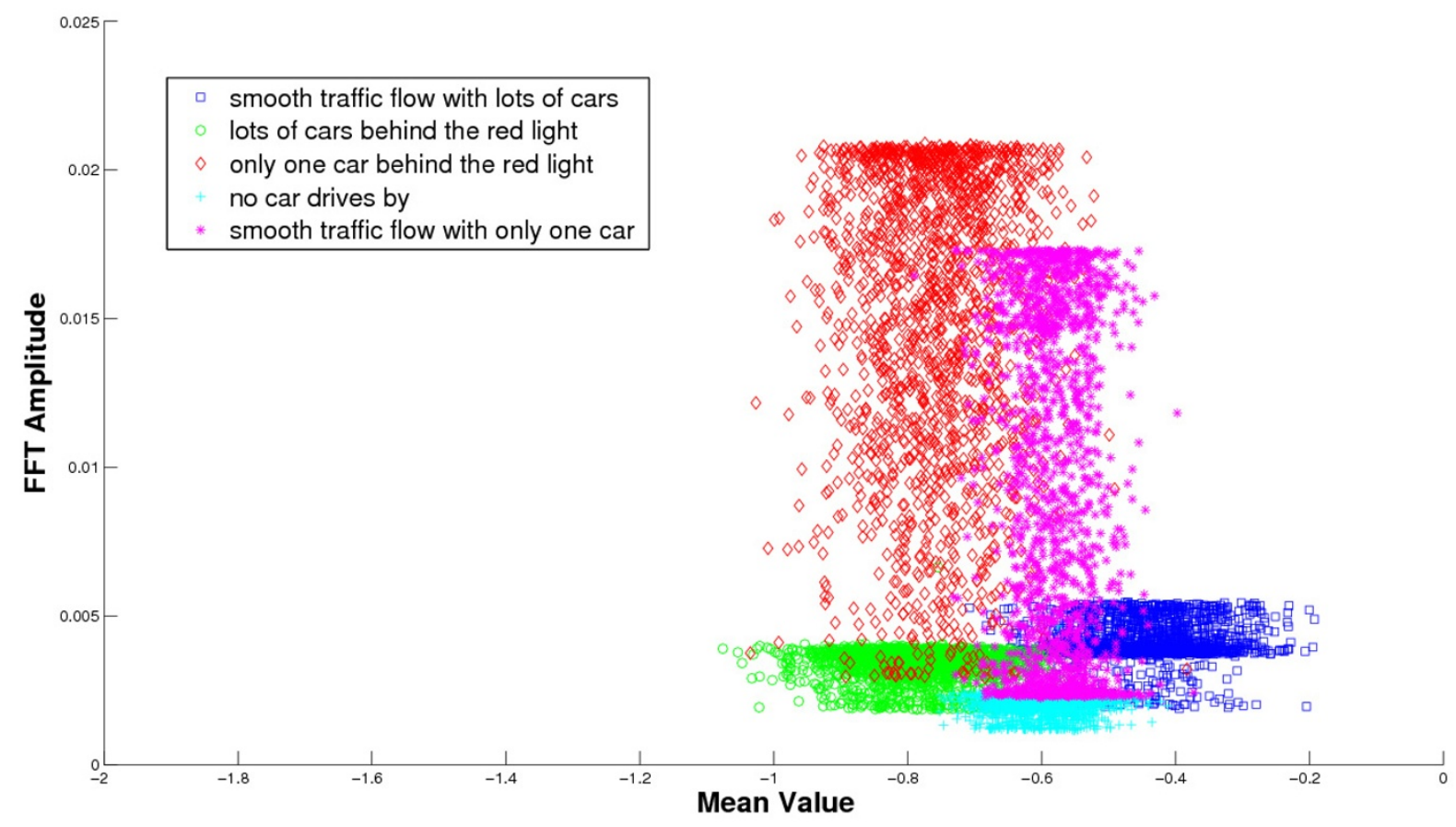

Figure 8 Classification results of five different traffic situations using decision tree learning (regression trees). $X$ and $Y$-axis are mean value and FFT amplitude of the RF-signals respectively, which are two features of the context classification process. 

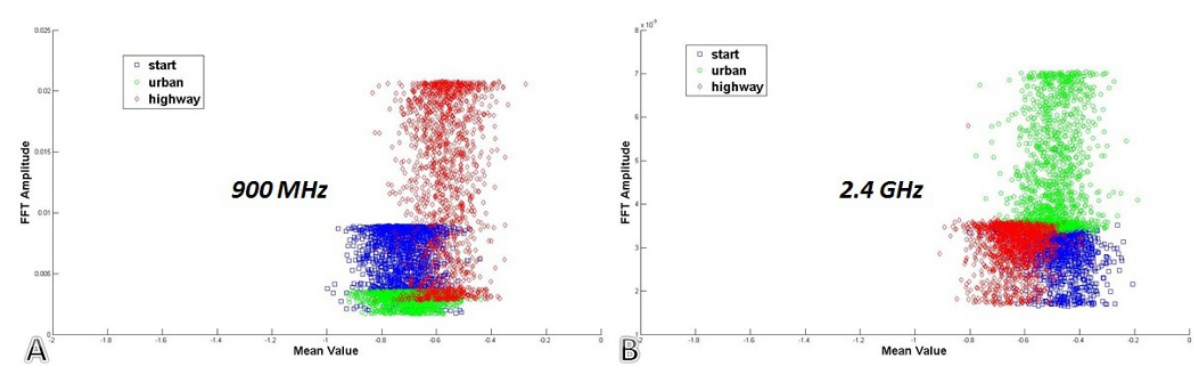

Figure 9 Classification results of three different location scenarios using decision tree learning (regression trees). $X$ and $Y$-axis are mean value and FFT amplitude of the RF-signals respectively, which are two features of the context classification process. (a) RF receiving at 900 $\mathrm{MHz}$; (b) RF receiving at $2.4 \mathrm{GHz}$.

Table 10 Time statistics of feature extraction process and individual classification algorithms (in s)

\begin{tabular}{ll}
\hline Process & Elapsed time pro run \\
\hline Feature extraction & 5.450 \\
Decision tree & 0.421 \\
k-nearest neighbor & 10.311 \\
Naive Bayes & 14.372 \\
\hline
\end{tabular}

simple structure, and other than most of the previous RFbased context recognition methods, it does not need reflection of a certain signal from the vehicles. The signals are generated inside the motor during combustion, in the (oil or water) pumps, fans, and connections of the sensors to the processing unit. In the main experiment, the signals are received by a roadside receiver and classified to extract the traffic situation. The complementary experiment shows well detecting, where the car is.

To show the performance of the proposed technique, we focused on the traffic density, traffic flow, and vehicular location. For instance, our classifiers could detect five different classes of traffic situation: no car, no traffic congestion, light traffic congestion, light traffic jam, and heavy traffic jam. Different classifiers are tested and performances more than $95 \%$ are achieved.

Differing from the current traffic sensing techniques, we propose our system to work in parallel with current vision-based traffic monitoring techniques. Because of its novelty, the proposed technique has various potential extensions, such as recognition of various classes of vehicles, development of a traffic surveillance network based on multiple antennae or directional traffic sensing by directional or array antenna.

\section{Endnote}

${ }^{a}$ http://www.ettus.com.

\section{Abbreviations}

RF: radio frequency; ITS: intelligent transport system; RADAR: radio detection and ranging; C2C: car-to-car; WLAN: wireless local area network; GSM: global system for mobile communications; RSSI: received signal strength indicator; USRP: universal software radio peripheral; FFT: fast Fourier transform; RMS: root mean square; $k$-NN: $k$-nearest neighbor.

\section{Acknowledgements}

We would like to acknowledge funding by the German Research Foundation (DFG) in the project "SenseCast: Context Prediction for Optimization of Network Parameters in Wireless Sensor Networks" (BE4319/1) and "Emergent Radio: The Emergent Radio Resources for Collaborative Data

Communication" (as part of priority program 1183).

\section{Competing interests}

The authors declare that they have no competing interests.

Received: 18 July 2011 Accepted: 10 January 2012

Published: 10 January 2012

\section{References}

1. M Tomizuka, Automated highway systems-an intelligent transportation system for the next century, in IEEE/ASME International Conference on Advanced Intelligent Mechatronics '97, Tokyo, Japan, (16-20 June 1997), p. 1

2. K Robert, Video-based traffic monitoring at day and night: vehicle features detection and tracking, in 12th International IEEE Conference on Intelligent Transportation Systems, St. Louis, (4-7 Oct 2009), pp. 285-290

3. C Setchell, EL Dagless, Vision-based road-traffic monitoring sensor, in IEE Proceedings of Vision, Image and Signal Processing, vol. 148. (University of Bristol, Briston, 2001), pp. 78-84. doi:10.1049/ip-vis:20010077

4. Y Ding, B Banitalebi, T Miyaki, M Beigl, RFTraffic: passive traffic awareness based on emitted RF noise from the vehicles, in ITS Telecommunications (ITST) 2011 11th International Conference on, St. Petersburg, Russia, (2011), pp. 393-398

5. CM Bishop, Pattern Recognition and Machine Learning, (Springer, New York, 2006), 8

6. A Starzacher, B Rinner, Single sensor acoustic feature extraction for embedded realtime vehicle classification, in Parallel and Distributed Computing, Applications and Technologies, 2009 International Conference on, Higashi Hiroshima, (8-11 Dec 2009), pp. 378-383

7. H Bischof, M Godec, C Leistner, M Hennecke, A Maier, J Wolf, B Rinner, A starzacher, autonomous multi-sensor vehicle classification for traffic monitoring. Data Mobil AISC. 81, 15-26 (2010). doi:10.1007/978-3-64215503-1_2

8. JA Quinn, R Nakibuule, Traffic flow monitoring in crowded cities, in Proceedings of 2010 AAAl Spring Symposium Series, Standford, (2010), pp. 73-78

9. RYQ Lai, Online vehicle detection for estimating traffic status. CoRR abs/ 1107.1058. 5 (2011)

10. Kun A, Z Vamossy, Traffic monitoring with computer vision, in Applied Machine Intelligence and Informatics, 2009. SAMI 2009. 7th International Symposium on, Herlany Slovakia, (2009), pp. 131-134

11. K Robert, Night-time traffic surveillance: a robust framework for multivehicle detection, classification and tracking, in Advanced Video and Signal Based Surveillance, 2009. AVSS '09. Sixth IEEE International Conference, Genova, (2-4 April 2009), pp. 1-6 
12. S Feng, $L Y U, Y$ Hong, $C$ Hong-jie, Traffic event duration forecast on urban expressway base on decision tree, in Control Engineering of China, Shangai, China, (2010)

13. Z Yang, G Liqun, Y Shufan, A new method for tracing by using corner detecting and $\mathrm{k}$-nearest neighbor, in Intelligent Information Technology and Security Informatics, International Symposium, Jinggangshan, (2010), pp. $337-340$

14. C Fischer, B Tibken, T Fischer, Left behind occupant recognition in parked cars based on acceleration and pressure information using $k$-nearestneighbor classification, in Intelligent Vehicles Symposium (IV), 2010 IEEE, San Diego, CA, (21-24 June 2010), pp. 1242-1247

15. RH Güting, T Behr, J Xu, Efficient k-nearest neighbor search on moving object trajectories. VLDB J. 19, 687-714 (2010). doi:10.1007/s00778-0100185-7

16. W Kasprzak, An Iconic classification scheme for video-based traffic sensor tasks, in Proceedings of the 9th International Conference on Computer Analysis of Images and Patterns, CAIP '01, Springer, London, UK, (2001), pp. 725-732.

17. L Peihong, D Liya, L Jilin, A video-based traffic information extraction system, in IEEE Proceedings on Intelligent Vehicles Symposium, Hangzhou, China, (9-11 June 2003), pp. 528-532

18. B Tseng, L Ching-Yung, J Smith, Real-time video surveillance for traffic monitoring using virtual line analysis, in Conference on Multimedia and Expo, 2002. ICME '02. Proceedings. 2002 IEEE International, vol. 2. (Hawthorne, NX, 2002), pp. 541-544

19. JC Tai, ST Tseng, CP Lin, KT Song, Real-time image tracking for automatic traffic monitoring and enforcement applications. Elsevier J Image Vis Comput. 22(6), 485-501 (2004)

20. R Cucchiara, M Piccardi, P Mello, Image analysis and rule-baseds reasoning for a traffic monitoring system. IEEE Trans Intel Transp Syst. 1(2), 119-130 (2000). doi:10.1109/6979.880969

21. T Willke, P Tientrakool, N Maxemchuk, A survey of inter-vehicle communication protocols and their applications. IEEE Commun Surv Tutor. 11(2), 3-20 (2009)

22. T Nadeem, S Dashtinezhad, CY Liao, L Iftode, TrafficView: traffic data dissemination using car-to-car communication, in ACM SIGMOBILE Mobile Computing and Communications Review (MC2R), vol. 8. (New York, USA, 2004)(3)

23. M Baldauf, MS Dustdar, A survey on context-aware systems. Int J Ad-hoc Ubiquitous Comput. 2(4), 263-277 (2007). doi:10.1504/JAHUC.2007.014070

24. A Dey, G Abowd, Towards a better understanding of context and context awareness, in Technical report git-gvu-99-22, (College of Computing, Georgia Institute of Technology, 1999)

25. P Lee, W Seah, HP Tan, Z Yao, Wireless sensing without sensors-an experimental study of motion/intrusion detection using RF irregularity. Meas Sci Technol. 21(124007) (2010). 7

26. M Scholz, S Sigg, G Bagschik, T Guenther, G von Zengen, D Shishkova, Y Ji, M Beigl, SenseWaves: radiowaves for context recognition, in Video submission of Pervasive'11, San Fransisaco, CA, (12-15 June 2011)

27. K Woyach, D Puccinelli, M Haenggi, Sensor-less sensing in wireless networks: implementation and measurements, in Proceedings of the Second International Workshop on Wireless Network Measurement (WiNMee), University of Notre Dame, IN, (3-6 April 2006)

28. K Muthukrishnan, M Lijding, N Meratnia, P Havinga, Sensing motion using spectral and spatial analysis of WLAN RSSI, in Proceedings of the 2nd European conference on Smart sensing and context, EuroSSC'07, Springer, Berlin, Heidelberg, (2007), pp. 2-76.

29. T Sohn, A Varshavsky, A Lamarca, MY Chen, T Choudhury, I Smith, S Consolvo, J Hightower, WG Griswold, ED Lara, Mobility detection using everyday gsm traces, in Proceedings of the Eighth International Conference on Ubiquitous Computing (Ubicomp'06), Springer, Heidelberg, (2006), pp. 212-224

30. I Anderson, H Muller, Context awareness via gsm signal strength fluctuation, in Pervasive 2006, Late Breaking Results, (Dublin, Ireland, 2006), pp. 27-31

31. S Gupta, MS Reynolds, SN Patel, ElectriSense: single-point sensing using EMI for electrical event detection and classification in the home, in Proceedings of the 12th ACM international conference on Ubiquitous computing, (New York, 2010)

32. M Reschke, S Schwarzl, J Starosta, S Sigg, M Beigl, Context awareness through the RF-channel, in Proceedings of the 2nd workshop on ContextSystems Design, Evaluation and Optimisation, (Como, Italy, 2011)
33. X Dong, H Weng, D Beetner, T Hubing, D Wunsch, M Noll, H Goeksu, B Moss, Detection and identification of vehicles based on their unintended electromagnetic emissions. IEEE Trans Electromagn Compat. 48(4), 752-759 (2006)

34. A Ruddle, D Topham, D Ward, Investigation of electromagnetic emissions measurements practices for alternative powertrain road vehicles. IEEE Int Sym Electromagn Compat. 2, 543-547 (2003)

35. V Kastrinaki, M Zervakis, K Kalaitzakis, A survey of video processing techniques for traffic applications. Image Vis Comput. 21(4), 359-381 (2003). doi:10.1016/50262-8856(03)00004-0

36. L Bao, SS Intille, Activity recognition from userannotated acceleration data, in Pervasive Computing, (Vienna, Austria, 2004), pp. 1-17

37. E Welbourne, L Lester, A Lamarca, G Borriello, Mobile context inference using low-cost sensors, in Proceedings of LoCA, (Springer, New York, 2005), pp. 254-263

38. J Lester, T Choudhury, G Borriello, A practical approach to recognizing physical activities, in Proc of Pervasive 2006, (Dublin, Ireland, 2006), pp. 1-16

39. E Tapia, S Intille, W Haskell, K Larson, J Wright, A King, R Friedman, Realtime recognition of physical activities and their intensities using wireless accelerometers and a heart rate monitor, in Wearable Computers, 2007 11th IEEE International Symposium, (Boston, 2007), pp. 37-40

40. S Magnussen, RE McRoberts, EO Tomppo, Model-based mean square error estimators for $k$-nearest neighbour predictions and applications using remotely sensed data for forest inventories. Remote Sens Environ. 113(3), 476-488 (2009). doi:10.1016/j.rse.2008.04.018

41. D Trewartha, Investigating Data Mining in MATLAB, (Bachelor's thesis, Bachelor (Honours) of Science Thesis of Rhodes University, 2006)

42. W Dwinnell, Modeling methodology 5: mathematical programming languages. PC Al. 12, 18-20 (1998)

43. M Rahman, A Haniz, S Khadka, Iswandi, M Kim, J ichi Takada, Design and Implementation of Emergency Radio Information System, (Technical report of ieice, The Institute of Electronics, Information and Communication Engineers, 2009)

44. M Rahman, A Haniz, S Khadka, Iswandi, M Kim, J ichi Takada, Development of Spectrum Sensing System with GNU Radio and USRP to Detect Emergency Radios. leice technical report sr2009-57(2009-10), (The Institute of Electronics, Information and Communication Engineers, 2009)

45. H Wang, W Jouini, R Hachemani, J Palicot, LS Cardoso, M Debbah, Blind bandwidth shape recognition for standard identification using USRP platforms and SDR4all tools. Adv Int Conf Telecommun. 0, 147-152 (2010)

46. A Tabassam, F Ali, S Kalsait, M Suleman, Building software-defined radios in MATLAB simulink-a step towards cognitive radios, in Computer Modelling and Simulation (UKSim), 2011 UKSim 13th International Conference, UK, (30 March-1 April 2011), pp. 492-497

47. P Pawelczak, K Nolan, L Doyle, SW Oh, D Cabric, Cognitive radio: ten years of experimentation and development. Commun Mag IEEE. 49(3), 90-100 (2011)

48. JR Quinlan, C4.5: Programs for Machine Learning, (Morgan Kaufmann Publishers Inc., San Francisco, CA, 1993), p. 302

49. J Yang, Toward physical activity diary: motion recognition using simple acceleration features with mobile phones, in Proceedings of the 1st international workshop on Interactive multimedia for consumer electronics, IMCE '09, (ACM, New York, NY, USA, 2009), pp. 1-10

50. AG Bonomi, in Sensing emotions, Vol. 12 of Philips Research Book Series, ed. by J Westerink, M Krans, M Ouwerkerk (Springer, Netherlands, 2011), pp. 41-51. Physical Activity Recognition Using a Wearable Accelerometer

51. JR Kwapisz, GM Weiss, SA Moore, Activity recognition using cell phone accelerometers. SIGKDD Explor Newslett. 12, 74-82 (2011). doi:10.1145/ 1964897.1964918

52. R Roiger, MW Geatz, Data Mining: A Tutorial Based Primer, (Addison Wesley, USA, 2003), p. 350

53. N Friedman, D Geiger, M Goldszmidt, Bayesian network classifiers. Mach Learn. 29(2/3), 131-163 (1997). doi:10.1023/A:1007465528199

54. T Cover, P Hart, Nearest neighbor pattern classification. IEEE Trans Inf Theory. 13, 21-27 (1967)

55. SY Sohn, Meta analysis of classification algorithms for pattern recognition. IEEE Trans Pattern Anal Mach Intel. 21(11), 1137-1144 (1999). doi:10.1109/ 34.809107

56. A Secker, MN Davies, AA Freitas, J Timmis, M Mendao, DR Flower, An experimental comparison of classification algorithms for the hierarchical 
prediction of protein function. BCS-SGAI Mag (Expert Update). 9(3), 17-22 (2007)

57. IH Witten, E Frank, MA Hall, Data Mining: Practical Machine Learning Tools and Techniques, (Morgan Kaufmann, San Francisco, 2011), 3, pp. 85-145

58. TA Milligan, Modern Antenna Design, (Wiley, Hoboken, 2005), 2, p. 614

59. LV Trees, Detection, Estimation and Modulation theory, Part IV, Optimum array processing, (Wiley, New York, 2002)1, , p. 1456

doi:10.1186/1687-1499-2012-8

Cite this article as: Ding et al:: RFTraffic: a study of passive traffic

awareness using emitted RF noise from the vehicles. EURASIP Journal on Wireless Communications and Networking 2012 2012:8.

\section{Submit your manuscript to a SpringerOpen ${ }^{\mathcal{O}}$ journal and benefit from:}

- Convenient online submission

- Rigorous peer review

- Immediate publication on acceptance

- Open access: articles freely available online

- High visibility within the field

- Retaining the copyright to your article

Submit your next manuscript at $\gg$ springeropen.com 